\title{
Effects of Ce on As-cast and Extrusion Microstructure of ZM21 Magnesium Alloy
}

\author{
Quan $\mathrm{Li}^{1, a_{*}}$, Weibo Zhu ${ }^{1}$, Aimin Jiang ${ }^{1}$, Bin Zeng ${ }^{1,2}$ \\ ${ }^{1}$ Chongqing Academy of Science and Technology, Chongqing 401123, China \\ ${ }^{2}$ College of Materials Science \& Engineering, Chongqing University, Chongqing 400030, China \\ a156723583@qq.com \\ Corresponding Author: Quan Li
}

Keywords: Magnesium alloy; Homogenization; Microstructure; Extrusion

\begin{abstract}
In this paper, the effects of Ce addition on as-cast and extrusion alloy microstructure of ZM21 Magnesium Alloy were investigated by the use of OM. Analyses the influence of ZM21 magnesium alloy as-cast and regularity and mechanism of the influence of extrusion organization, the results show that rare earth Ce can effectively refine ZM21 magnesium alloy grain organization, and can improve the mechanical properties such as plasticity and strength of the alloy.
\end{abstract}

\section{Introduction}

Magnesium alloy is not only the quality is light, but also has high than strength, stiffness, damping performance is good, strong ability to resist radiation, and a series of advantages, it is very important to the future of metal structural materials and functional materials ${ }^{[1-3]}$. Since the turn of the century, the countries all over the world to pay more attention to energy and environmental protection, research and development of magnesium in the new situation, magnesium alloys become the rapid rise of new engineering materials. By adding different content of cerium ZM21 magnesium alloy extrusion deformation experiments of cerium on the ZM21 magnesium alloy as-cast and extrusion research organization rule and mechanism of the influence of the optimization of rare earth element cerium in ZM21 magnesium alloy addition amount, in order to obtain excellent comprehensive mechanical properties of the alloy, and to provide experimental basis ${ }^{[4-5]}$.

\section{Experiments}

Table 1. Chemical component of the alloy ( wt\%)

\begin{tabular}{ccccc}
\hline \multirow{2}{*}{ Alloy } & \multicolumn{4}{c}{ Alloy chemical composition } \\
Number & Zn & Mn & Ce & Mg \\
\hline 1\# & 1.80 & 0.95 & 0 & other \\
2\# & 1.92 & 0.91 & 0.19 & other \\
3\# & 2.12 & 0.93 & 0.28 & other \\
4\# & 2.06 & 0.92 & 0.39 & other \\
5\# & 2.14 & 0.91 & 0.57 & other \\
\hline
\end{tabular}

Alloy raw materials including $\mathrm{Mg}$ (mass fraction 99.3\%), Zn (mass fraction 99.9\%), 0.39 Mg-Mn intermediate alloy and Mg-20Ce intermediate alloy, with low carbon steel crucible in 60 $\mathrm{kW}$ resistance furnace refining, 5 flux as refining agent and coating agent, by a water-cooled semicontinuous casting system cast into casting of $\Phi 92 \mathrm{~mm}$. Melting and pouring process with $\mathrm{CO}_{2}$ and $\mathrm{SF}_{6}$ gas mixture for their own protection. By $\mathrm{Mg}$ successive increase in 2.0 recent $1.0 \mathrm{Mn}$ alloy Mg-20Ce intermediate alloy, access to Mg-2.0Zn-1.0Mn for the substrate, the laser spectrometer detection component as shown in table 1 . Will cast the sample in the $12 \mathrm{kw}$ box-type resistance furnace of wind circulation homogenization processing, $12 \mathrm{~h}$ under $420^{\circ} \mathrm{C}$ temperature annealing; And then, in 500 tons of extrusion production test horizontal extrusion machine, $400^{\circ} \mathrm{C}$ preheat $2 \mathrm{~h}$, extrusion temperature is $400^{\circ} \mathrm{C}$, extrusion ratio for 28 , extrusion products $\Phi 16 \mathrm{~mm}$ diameter. 
Squeezed state sample direct interception on extrusion rods, picric acid solution after corrosion, watch a OPTEC company MDS metallographic microscope.

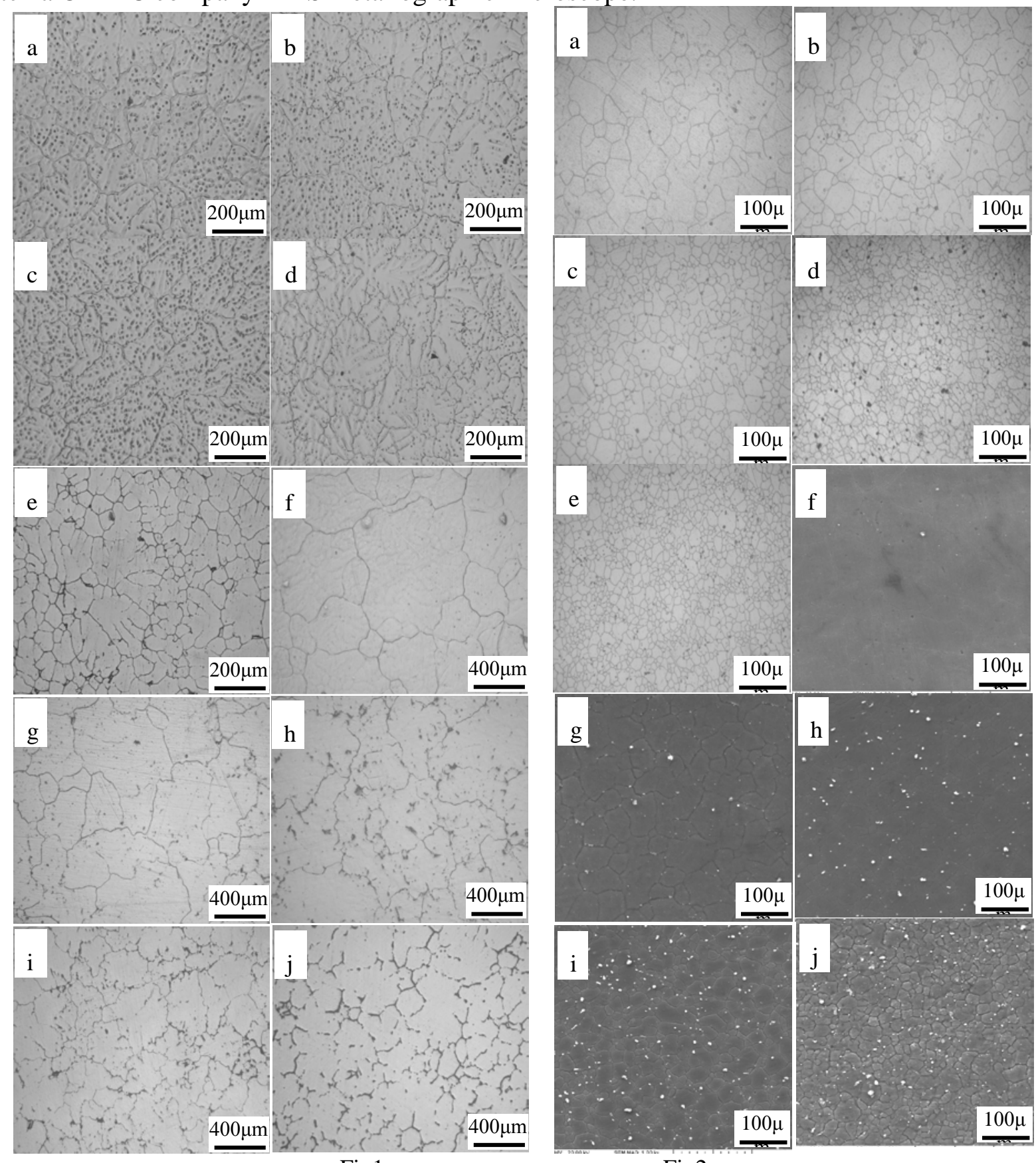

Fig1

Fig2

Fig1 microstructure of as cast and homogenized alloy

(a) cast, 1\# ; (b) cast, 2\#; (c) cast,3\#; (d) cast, 4\#; (e) cast, 5\#; (f) Homogenized, 1\#; (g) Homogenized, 2\#;

(h) Homogenized, 3\#; (i) Homogenized, 4\#; (j) Homogenized, 5\#

Fig. 2 Optical microstructures and SEM images of extruded alloy

(a) Optical microstructure, 1\#; (b) Optical microstructure, 2\#; (c) Optical microstructure,3\#; (d) Optical microstructure, 4\#; (e) Optical microstructure, 5\#; (f) SEM image, 1\#; (g) SEM image, 2\#; (h) SEM image, 3\#; (i) SEM image, 4\#; (j) SEM image, 5\#

\section{Test results and analysis}

Figure 1 is adding different content of Ce ZM21 magnesium alloys as-cast and homogenization tissue. 1 \# alloy secondary dendrite is developed; 2 \# alloy because of adding trace elements Ce, alloy secondary dendrite gaps appear grayish fine second phase, consisting of a dendrite gap larger 
than 1 \# alloy reticular structure, and distribution of discontinuous shallow bright color compounds, shows that Ce compounds tend to be in a dendrite partial together; 3 \# alloy secondary dendrite clearance in the second phase, a dendritic clearance is fine mesh, the mesh structure compared with 1 \# and 2 \# alloy obviously refined, the secondary dendrite arm spacing of the tiny; 4 \# alloy mesh structure refinement, there is a small amount of secondary dendrite arm space bright color of the second phase particles, the secondary dendrite arm spacing relative to the 3 \# alloy become bigger; 5 \# alloy compounds are mainly distributed in a dendrite gaps, formed a continuous mesh structure, mesh structure further refinement, but cable due to the partial polymer more coarsens, second time compared with 4 \# alloy further dendrite arm spacing. As-cast organization after homogenizing annealing, the microstructure varying degrees of changes have taken place. In 1 \# and 2 \# alloy, the dendritic segregation has completely disappeared, as-cast dot compounds have been dissolved in the matrix in the organization, and organization boundary obviously. In 3 \# alloy, the dendritic segregation is basically eliminated, but there are still a small amount of residue. After homogenizing annealing, intercrystalline compound of 4 \# and $5 \#$ alloy get partly dissolved, get eliminate dendritic morphology, matrix composition tend to be more uniform.

Figure 2 is adding rare earth Ce ZM21 magnesium alloy extrusion state of organization and the SEM topography, extrusion, after adding rare earth Ce alloy obviously refined grains, along with the increase of the content of Ce alloy grain refinement gradually, this is mainly due to the effect of rare earth Ce has fine grains on the one hand, the formation of intermetallic compound cerium and magnesium Mg - Ce phase for high melting point compounds, distributed in the intracrystalline and grain boundary, hinder the grain boundary migration, and tiny recrystallization grain size is obtained. In addition, in the second phase and the dispersion of the surrounding, squeeze the apparent cause distortion, distortion area with high dislocation density and the relatively large difference of grain boundary orientation, distortion region become the core of recrystallization, increases the nucleation rate, so that the grain refinement. Compared with 1 \# alloy, intergranular of 2 \# alloy are obviously fine recrystallization grain size; Grain than 2 \# 3 \# alloy alloy obviously small, recrystallization grain size proportion will increase, there is almost no special big grain; Than 3 \# 4 \# alloy has a further refinement, recrystallization grain size more small; Relative to the 4 \# alloy, 5 \# alloy grain refinement effect is no longer apparent. The SEM morphology of alloy extrusion states shows that 1 \# 5 \# alloy substrate has a granular in the second phase, in which the number of the second phase in 1 \# alloy minimum, along with the increase of the content of rare earth Ce gradually increase the number of the second phase, grain size became smaller at the same time; Second phase in the alloy 1 \# and 2 \# alloy particles and the alloy grain size is smaller, and 5 \# 4 \# alloy in the second phase and the grain size is larger, according to the statistics, 2 \# alloy grain size is about $20 \sim 35$ um, 3 \# alloy grain size is about $15 \sim 25$ um, 4 \# alloy grain size of about 8 to $15 \mathrm{um}$, \# 5 grain size is about $5 \sim 12 \mathrm{um}$, the second phase particle size is about $2 \sim 3 \mathrm{um}$. 3 \# and 5 \#, 4 \# alloy alloy in the second phase distribution of the relative dispersion, but 5 \# alloy of second phase particles than 3 \# and 4 \# alloy alloy particle size.

\section{Conclusions}

After extrusion, add Ce elements of ZM21 magnesium alloy obviously refined grains, along with the increase of the content of Ce alloy grain refinement gradually, but when the Ce content reaches $0.39 \%$, the refining effect is stable, even add Ce to $0.57 \%$, no significant effect of grain refinement.

\section{Acknowledgements}

This work is supported by the International Science and Technology Cooperation Project (2013DFA71070 、 2014DFG52810); Chongqing integrated demonstration projects (cstc2013jcsf50003); 


\section{References}

[1] J.S. Zhang, J. Yan, W. Liang, C.X. Xu, C.L. Zhou. Icosahedral quasicrystal phase in Mg-Zn-Nd ternary system [J]. Materials Letters, 62 (2008): 4489-4491.

[2] Yu Kun, Li Wenxian, Zhang Shijun. Mechanism of Grain Refining by Adding Cerium in Mg and Mg Alloys[J]. Rare Metal Materials and Engineering, 2005, Vol.34(7):1013-1016.

[3] Ravi Kumar N V, Blandin 1 J, Desrayaud C et al. Grain refinement in A791 magnesium alloy during thermomechanical processing(J]. Materials Science and Engineering, 2003, A359: 150-157.

[4] Bussiba A, Ben Artzy A, Shtechman A et al. Grain refinement of AZ31 and ZK60 Mg alloys-towards superplasticity studies[J]. Materials Science and Engineering, 2001, A302: 56-62.

[5] Li Wenxian. Magnesium and magnesium alloys[M]. Changsha:Central South University Press, 2005.6. 